\title{
Philosophiques
}

\section{Linguistique, philosophie du langage et épistémologie (Réponse à P. Martin)}

\section{Ghyslain Charron}

Volume 3, numéro 2, octobre 1976

URI : https://id.erudit.org/iderudit/203056ar

DOI : https://doi.org/10.7202/203056ar

Aller au sommaire du numéro

Éditeur(s)

Société de philosophie du Québec

ISSN

0316-2923 (imprimé)

1492-1391 (numérique)

Découvrir la revue

Citer ce document

Charron, G. (1976). Linguistique, philosophie du langage et épistémologie

(Réponse à P. Martin). Philosophiques, 3(2), 261-278.

https://doi.org/10.7202/203056ar d'utilisation que vous pouvez consulter en ligne.

https://apropos.erudit.org/fr/usagers/politique-dutilisation/ 


\section{LINGUISTIQUE, PHILOSOPHIE DU LANGAGE ET EPISTÉMOLOGIE (RÉPONSE À P. MARTIN) par Ghyslain CHARRON}

Martin n'en finit pas de rappeler que Merleau-Ponty se fourvoie au sujet de Saussure, que "la méconnaissance et l'utilisation fautive des concepts linguistiques sont à l'origine du fossé qui sépare encore aujourd'hui savants et philosophes". Mon livre voulait uniquement faire ressortir la spécificité de l'analyse fonctionnelle et structurale de Martinet et celle de l'approche phénoménologique de Merleau-Ponty, montrer leur cohérence en rattachant l'ensemble des concepts fondamentaux aux postulats et aux définitions de base. Je lui avouerai qu'en lisant son étude critique, je me trouve plus en face de ses hantises que du contenu de mon livre. Au lieu d'analyser et de discuter les thèses essentielles que j'avance, il lance pêle-mêle des remarques qui pour la plupart me paraissent non pertinentes si l'on tient compte du contexte.

D'abord, ce n'est pas un "résumé " de la pensée de Martinet que je proposais au lecteur. Je n'ai jamais cru qu'on pouvait décemment résumer en cinquante pages quatre ou cinq mille pages d'études détaillées de phénomènes linguistiques déterminés'. J'ai seulement essayé de repérer l'appareil conceptuel et théorique construit par Martinet (1ère partie de mon livre) pour ensuite expliciter les présuppositions et les conséquences et les mettre en contraste avec celles de Merleau-Ponty (3ème partie). Mon idée à moi était que "la façon selon laquelle Martinet et Merleau-Ponty lisent Saussure et s'en inspirent [...] présuppose et manifeste la spécifi-

1. Martin estime que le non-linguiste qui s'en tiendrait à ma présentation de la linguistique de Martinet n'en aurait " qu'une idée bien abstraite ". J'en conviens aisément. Il n'y a qu'un moyen pour se faire une "idée concrète "du travail du linguiste "sur le terrain" : c'est de travailler sur le terrain, c'est-à-dire décrire des langues. 
cité de leur projet respectif $\aleph^{2}$. Ma préoccupation n'était pas de mesurer le degré de "fidélité " que l'un et l'autre entretenaient à l'égard de Saussure mais de déceler la perspective de chacun par la façon même dont ils parlaient de Saussure, s'en servaient en le transformant et en le critiquant. Tout le monde sait depuis longtemps que la "fidélité " à la lettre et la répétition dévote n'ont jamais été le fort de Merleau-Ponty. Ce n'est pas seulement parce qu'il ne pratiquait pas assez la linguistique que Merleau-Ponty a interprété Saussure de cette façon. Il a fait la même chose avec Kant ou Husserl, avec Descartes, Marx ou Freud. On ne peut utiliser l'œuvre des autres sans l'interpréter. Pour lui, il n'y a pas à choisir entre "l'interprétation objective et l'arbitraire". Interpréter, c'est penser l'impensé de l'auteur et, bien sûr, pour le faire, utiliser ses idées à soi. "Si l'on croit que l'interprétation est astreinte ou à déformer ou à reprendre littéralement, c'est qu'on veut que la signification d'une ouvre soit toute positive, et susceptible en droit d'un inventaire qui délimite ce qui y est et ce qui n'y est pas. "(Signes, p. 202). Et Saussure lui-même ne savait pas toujours clairement où il en était ; il n'aurait pas été capable d'un tel inventaire. Sur une opposition considérée aussi fondamentale que celle entre langue et parole, Martinet estime qu'il " n'est pas sûr que Saussure lui-même s'y retrouve toujours " ${ }^{3}$. Et combien de fois encore faudra-t-il répéter que Merleau-Ponty en s'inspirant de Saussure ne faisait pas d'abord un travail d'historien de la linguistique $^{4}$ ! Il tâchait plutôt d'élaborer sa philosophie de la parole. II utilisait les textes de Saussure, de Wartburg, de Pos, etc., pour for-

2. G. CHARRON, Du Langage, p. 149. N'est-ce pas à partir et en fonction de la phonologie que Martinet juge Saussure? "Mais Saussure, dit-il, ne fait jamais le pas décisif qui fondera la phonologie en reconnaissant expressément au phonème un statut linguistique. Les linguistes qui, aujourd'hui, se déclarent saussuriens sont souvent ceux qui n'ont pas compris la nature de la révolution phonologique. "( $A$. MARTINET, $F$. de Saussure dans Encyclopecdia Universalis, vol. 14, p. 696). "L'enseignement saussurien a fait faire à la recherche un pas décisif en distinguant absolument entre synchronie et diachronie. Mais la dichotomie langue-parole, en renforçant le psychologisme traditionnel, n'a pu que retarder l'avènement de la phonologie et, lors même que celle-ci s'était établie, elle a longtemps empêché certains linguistes de reconnaittre les véritables fondements de la nouvelle discipline. " (A. MARTINET, "Les choix du locuteur " dans Revue Pbilosophique de la France et de l'Étranger, 1966, no 3, p. 273).

3. A. MARTINET, "F. de Saussure ", dans Encyclopcedia Universalis, vol. 14, p. 696.

4. D'ailleurs, même l'historien ne peut aborder son objet d'étude d'un regard vierge de toute idée et du point de vue de Sirius ; comme tout le monde, il parie à partir d'un 
muler sa propre pensée. Celui qui fait l'étude critique d'un ouvrage ou entreprend son édition critique a d'autres préoccupations, il va sans dire. Or, ce même Martin pour qui les faits semblent sacrés a trouvé le moyen de passer sous silence tous les arguments principaux de mon livre. Il a cru plus important d'entonner une fois de plus le refrain si souvent chanté par Martinet ou Mounin à l'endroit de Merleau-Ponty, de Lévi-Strauss, de Lacan, de Barthes, d'Althusser, etc : "utilisation fautive" des concepts de la linguistique. Je ne m'attarderai pas, dit Martin, à l'exposé des théories de Martinet et de Merleau-Ponty et à la caractérisation de leurs postulats respectifs spécifiques. Il ne prend même pas la peine de discuter l'explicitation des présupposés que je propose des hypothèses et des concepts clés des deux auteurs. Pas un mot de ma discussion du principe d'économie chez Martinet, pas un mot sur ma critique de l'identification de la langue à un code, rien sur le concept de discours que j'invoque à la fin pour marquer les limites de la théorie de Martinet et de la phénoménologie de la parole de Merleau-Ponty, rien sur la distinction entre la signification comme intention signifiante et la signification (ou signifié) comme différence. Pour Martin, tout cela ne mérite pas d'être discuté ni même mentionné. Il note plutôt : "l'auteur n'a pas su tirer la conclusion théorique qui s'imposait : le dialogue de sourds entre linguistes et philosophes [...] ne tient pas tant à la spécificité des points de vue de chacun qu'à une mauvaise utilisation des concepts qui connaissent à l'intérieur de chaque discipline une utilisation particulière. "Qu'est-ce qu'une "mauvaise " utilisation? Si utiliser correctement un terme scientifique, c'est lui conserver dans le discours philosophique exactement la même valeur qu'il a dans le discours scientifique, il ne peut y avoir un usage philosophique absolument correct d'un terme scientifique. La valeur d'un terme, on le sait,

lieu où il se trouve et en fonction duquel se constitue sa perspective. Comme l'écrit R. Blanché : "Toute histoire, a-t-on dit, est contemporaine. Nä̈vement ou consciemment, nous projetons sur le passé, pour l'interpréter ou simplement pour le percevoir, non seulement nos connaissances nouvelles, mais aussi et surtout nos intérêts présents et notre outillage conceptuel du moment. De quoi l'histoire de la logique, on le verra, nous offre une bonne illustration. Le renouveau de cette discipline à notre époque a modifié notre regard, et il n'est plus possible aujourd'hui de voir la logique d'Aristote, celle des stoïciens, celle des médiévaux, et même celle des modernes de Leibniz à Boole inclusivement, du même œil dont on les regardait encore au début de notre siècle. L'histoire de la logique est à récrire, et l'on s'y emploie de divers côtés depuis quelques décennies. "(R. BLANCHE, La logique et son bistoire, d'Aristote à Russell, A. Colin, Coll. U, 1970, p. 5) 
tient aux relations et aux oppositions avec les autres termes du système et du discours où il trouve place et fonction. Qui croira que le terme parole, par exemple, a dans le langage ordinaire, chez Saussure, chez Merleau-Ponty et chez Martinet, absolument la même valeur! Quand Merleau-Ponty écrit : "Si mes paroles ont un sens, ce n'est pas parce qu'elles offrent l'organisation systématique que dévoilera le linguiste, c'est parce que cette organisation, comme le regard, se rapporte à elle-même ", peut-être tient-il du point de vue du linguiste un discours aberrant ou vide de sens, il montre en tout cas que son discours sur le langage n'est pas un double ou une copie conforme de celui du linguiste. Face au discours du linguiste, le philosophe peut ou s'inspirer des concepts qu'il y trouve et en faire des idées philosophiques pour formuler ses propres dévouvertes, ou expliciter les présuppositions des concepts, hypothèses et postulats du linguiste. De son côté, le linguiste peut s'inspirer de certaines idées philosophiques et les transformer en hypothèses ou concepts scientifiques. Un emprunt par une discipline des concepts d'une autre discipline me semble être toujours inévitablement une métamorphose, puisque l'unité empruntée est inscrite dans un nouveau système et elle est utilisée en vue d'un discours original. Quel intérêt y aurait-il, par exemple, à reprocher à Freud de n'avoir pas conservé aux termes inconscient, moi, traumatisme, transfert, sexualité, etc., le sens que ces termes avaient dans la philosophie ou dans la psychologie, dans la médecine, la chirurgie ou dans la langue commune. Le paradoxe justement est que tout emprunt est conservation et transformation. Conservation, c'est pourquoi on préfère employer un terme déjà en usage au lieu

5. Et Merleau-Ponty continue : "Comme il y a une réversibilité du voyant et du visible, et comme au point où se croisent les deux métamorphoses naît ce qu'on appelle perception, de même il y a une réversibilité de la parole et de ce qu' elle signifie ; la signification vient sceller, clore, rassembler la multiplicité des moyens physiques, physiologiques, linguistiques de l'élocution, les contracter en un seul acte, comme la vision vient achever le corps esthésiologique. "(V.I., p. 202). Je commentais dans mon livre cette proposition importante de la façon suivante: "Bref, si la structure est condition nécessaire de la signification, elle n'est pas condition suffisante. La signification existe bien sûr comme différence par rapport à toutes les autres significations mais elle est différence par l'acte du sujet parlant, par l'intention et la réflexion qui animent et orientent cet acte. "(G. CHARRON, Du langage, pp. 172-173). C'est là à mon avis la thèse essentielle de la philosophie de la parole de Merleau-Ponty et cette thèse montre assez que malgré son intérêt pour Saussure et le structuralisme, Merleau-Ponty ne renonce aucunement au point de vue phénoménologique. 
d'en créer un de toute pièce, métamorphose car le terme sera utilisé en vue d'exprimer une découverte et prendra un sens en partie nouveau. L'usage est justifié s'il est efficace, fécond, cohérent dans le système et le discours qui empruntent.

Lagueux, Madison et d'autres ont reconnu que l'interprétation de Saussure par Merleau-Ponty "est plutôt philosophique qu'historique ". Ça me paraît indiscutable. Je ne croyais pas nécessaire dans mon livre de m'appesantir sur ce fait. J'étais plus intéressé à reconstituer la perspective de Merleau-Ponty qu'à repérer dans le détail toutes les "erreurs" qu'il a pu commettre à l'égard de Husserl, Saussure, Descartes, etc. Á mon avis, ce n'est pas tant parce que Merleau-Ponty a méconnu Saussure ou qu'il a fait des concepts de la linguistique un "mauvais " usage qu'entre Martinet et Merleau-Ponty le fossé, comme on dit, n’a pas été comblé ou surmonté d'un pont. C'est plutôt que leurs convictions épistémologiques, explicites ou implicites, étaient irréductibles et en conséquence irréductibles aussi leurs conceptions de la science, de la philosophie et des relations entre elles. Il y a quelques années, Martinet voyait dans la mode de la linguistique un "drame atroce " et en tenait Merleau-Ponty en partie responsable. Il a été le premier, dit Martinet, à lancer la mode, il " a cru pouvoir s'inspirer de Saussure sans avoir de la linguistique une connaissance sérieuse. Il s'est posé des problèmes qu'un linguiste s'interdirait d'aborder ". Depuis quand revient-il au linguiste de déterminer les questions qu'il est permis ou interdit au philosophe de poser? N'y a-t-il de légitimes, même pour le philosophe, uniquement les problèmes que les linguistes se permettent d'aborder! Le sourd ici, c'est celui qui déclare a priori que les seules questions permises sont les siennes. Dialogue de sourds aussi entre Martinet et Merleau-Ponty sur la "théorie " de la science: faire œuvre scientifique, estime Martinet, c'est "d'abord observer les faits"; Merleau-Ponty, lui, pense qu'il faut récuser avec la dernière énergie le "mythe du savoir scientifique qui attend de la simple notation des faits non seulement la science mais la science de cette science ". $\AA$ mon avis la principale conclusion à tirer, ce n'est pas que la " mauvaise utilisation " par Merleau-Ponty des concepts saussuriens explique le dialogue de sourds entre lui et Martinet, mais plutôt que leurs convictions épistémologiques et leurs présuppositions étaient irréconciliables. Merleau-Ponty a toute sa vie refusé le type de réalisme que professe Martinet. Je reviendrai sur ce point plus loin. Je voudrais 
d'abord discuter quelques-unes des remarques de détail mises en avant par Martin.

Quand il annonce que si le non-linguiste en reste à ma présentation de Martinet, il "ne pourra se faire qu'une idée bien abstraite [...] de la linguistique tout court ", je dois bien admettre : la linguistique tout court, je ne sais pas ce que c'est, pas une seconde il ne m'est venu à l'esprit l'idée d'instruire mon lecteur sur cette "linguistique tout court".

"On ne peut, dit Martin, passer sous silence les définitions bien incomplètes des unités distinctes et des unités significatives à la page 21. "Justement, il ne s'agissait pas dans ce paragraphe de donner une définition complète du phonème, ou unité distinctive, et du monème, ou unité significative, mais d'affirmer que la méthode de Martinet est une analyse fonctionnelle. Déjà aux pages 16-17, une citation de Martinet avait suffisamment déterminé pour ce chapitre préliminaire ce qu'il fallait entendre par phonème et monème. Les chapitres II (pp. 25-38) et III (pp. 39-52) sont justement consacrés à ces questions. Si Martin veut des définitions plus exhaustives, ce n'est pas à la page 21 qu'il faut les chercher mais aux pages 28 à 38, dont le titre me paraît assez évocateur : "Identification et définition des phonèmes ", et aux pages 39 à 43 intitulées "Détermination et hiérarchisation des monèmes".

Puis on m'avertit que les linguistes accepteraient difficilement une formulation comme celle-ci : " tout acte de parole est le résultat de vibrations de cordes vocales" (p. 35) m'objectant les consonnes sourdes et les voyelles chuchotées. Rappelons que cette proposition ouvre un paragraphe où il est question de la mélodie du discours, c'est-à-dire de la montée et de la descente de la voix dans l'énoncé. Ce que j'appelle là acte de parole n'est rien d'autre que l'énonciation d'un ou de plusieurs énoncés. Je n'ai aucunement affirmé que tous les phonèmes pris un à un impliquaient des vibrations des cordes vocales. Cette citation est tirée d'une section où il est question non des phonèmes mais des faits prosodiques ou suprasegmentaux. Je faisais allusion à la proposition de Martinet: "On classe dans la prosodie tous les faits de parole qui n'entrent pas dans le cadre phonématique, c'est-à-dire ceux qui échappent [. . . ] à la deuxième articulation. Physiquement, il s'agit en général des faits phoniques nécessairement présents dans tout énoncé parlé : que l'énergie avec laquelle on articule soit considérable ou 
limitée, elle est toujours là à un degré quelconque ; dès que la voix se fait entendre, il faut bien que les vibrations de la glotte aient une fréquence, ce qui donne à chaque instant, aussi longtemps que la voix est perçue, une bauteur mélodique déterminée ${ }^{6}$. "Comment s'organise le travail du larynx, de la glotte et des cordes vocales pour les deux fonctions linguistiques que sont l'accent et l'intonation, je laisserai aux phonéticiens et aux physiologistes le soin de le déterminer. Je sais seulement que des énoncés sans accent, sans intonation, sans voyelle et sans consonne sonore, je n'ai pas, Dieu merci, à en déchiffrer trop souvent dans la même journée !?

Si j'écris : "l'ordre selon lequel les monèmes s'articulent dans la chaîne n'est pas fortuit, mais est partiellement déterminé par la syntaxe de la langue " (p. 39), il s'empresse de me corriger : "il est prouvé, dit-il, qu'il n'en va pas de même dans toutes les langues". Peut-être bien en effet existe-t-il des langues sans aucune règle quant à la position des monèmes dans la chaîne parlée; je n'en sais rien, n'ayant pas examiné les trois mille et quelques langues passées ou présentes. Je sais cependant ce que j'avais en tête quand j'ai écrit la proposition que me reproche Martin, rien de plus et rien de moins que ce que dit Martinet dans l'énoncé suivant: "la position respective des monèmes est souvent déterminée par la tradition ou par le besoin de distinguer entre des énoncés ayant des sens différents : il se trouve que l'on ne dit pas Paul c'est avec allé que je Rome à suis et il est loin d'être indifférent que je fasse allusion à un grand bomme ou à un bomme grand, sans parler évidemment de la différence entre l'bomme tue l'ours et l'ours tue l'bomme. ${ }^{8} \mathrm{Il}$ y a quelques semaines, dans un

6. A. MARTINET, Éléments de linguistique générale, A. Colin, U prisme, 1974, p. 83.

7. "On nomme consonne, dit Martinet, les sons qui se perçoivent mal sans le soutien d'une voyelle précédente ou suivante. " (Ibid., p. 45). "La voix résulte de vibrations de cordes vocales [...]." "Comme les cordes vibrent à chaque instant à une hauteur déterminée, on peut pour tout énoncé, tracer une courbe des hauteurs mélodiques [...]. " (Ibid., p. 84).

8. A. MARTINET, Langue et Fonction, p. 62. "Dès que tout énoncé, dit-il plus loin, en quelque langue que ce soit, est conçu comme une succession de signes minimaux ... il devient évident que la grammaire peut être présentée comme l'ensemble des règles qui restreignent la libre combinaison de tous ces signes." (Ibid., p. 140). "Une langue dans laquelle tous les monèmes seraient du type bier, c'est-à-dire doués de l'autonomie syntaxique, parce que l'indication de la fonction fait partie du sens du terme, serait si peu économique que nous pouvons l'éliminer comme pratiquement impossible. " (Ibid., p. 92). "La relation entre deux éléments de l'énoncé peut ... être exprimée au moyen des positions respectives de leurs équivalents linguistiques ..." (Ibid., p. 83). 
ouvrage dirigé par le linguiste $M$. Mahmoudian, il m'est arrivé de lire ce qui suit : "Admettre que tout énoncé est une suite de monèmes nous place devant une alternative : ou bien les monèmes peuvent être assemblés librement dans les énoncés, ou bien la combinaison des monèmes est soumise à des restrictions. On a pu observer que la combinaison libre des monèmes n'est attestée dans aucune langue. [...] Dire que les monèmes sont soumis à des règles dans leur combinaison, c'est admettre qu'il y a une structure de la première articulation. ${ }^{9}$ Il va sans dire que le philosophe n'a pas compétence pour arbitrer le désaccord entre le linguiste Martin et les linguistes de l'équipe Mahmoudian.

Merleau-Ponty, dit Martin, emploie "de façon impropre" les termes signe et signification. Je dirais plutôt qu'il conserve la plupart du temps la terminologie du langage ordinaire où signe correspond à peu près à ce que Saussure appelle signifiant et signi. fication à signifié. Qu'on lise donc Merleau-Ponty avec cette hypothèse: "la fameuse définition du signe comme "diacritique, oppositif et négatif " veut dire que la langue est présente au sujet parlant comme un système d'écarts entre signes et entre significations [...]. ${ }^{10}$ Martin, lui, préfère chicaner sur l'usage du terme "diacritique "1. Il aurait pu s'apercevoir que Merleau-Ponty relevait ce qui lui paraissait l'essentiel de l'apport saussurien, à savoir

9. Dans Pour enseigner le Français, direction : M. MAHMOUDIAN, P.U.F., 1976, PP. 74-75.

10. MERLEAU-PONTY, Résumé de cours, p. 33. Quant au terme de "signification "Martin sait comme moi que Saussure lui-même continue de l'employer mais est loin d'être au clair sur les rapports entre signification, signifié et valeur. "La valeur, dit-il, prise dans son aspect conceptuel, est sans doute un élément de la signification et il est très difficile de savoir comment celle-ci s'en distingue tout en étant sous sa dépendance. "(Cours, p. 158). Si l'on dit que le rapport de signification est la relation unissant le signifiant au signifié, on pourra comme le souligne Martin après Saussure, en faire un "fait positif ". Tandis que le signifié tient sa valeur de ses relations aux autres signifiés du système. Je me contenterai de renvoyer ici aux interprétations de Burger, Godel. Prieto et au résumé de ces discussions que $T$. de Mauro présente dans son édition critique du Cours, pp. 464465. Quant aux fameuses propositions: "dans la langue il n'y a que des différences sans termes positifs "; "le mécanisme linguistique roule tout entier sur des identités et des différences ", Buyssens a vu entre elles deux thèses contradictoires. Ce sur quoi H. Frei n'est pas d'accord avec Buyssens. Pour cette controverse, voir Cabiers Ferdinand de Saussure 8, 1949, pp. 37-60; 9, 1950, pp. 7-28; 10 . 1952, pp. 47.50 .

11. Il n'est pas difficile de comprendre que Merleau-Ponty prend "diacritique "dans son sens étymologique : du grec diakrinein, distinguer, discriminer. 
la notion de valeur et de système. Les expressions "signe diacritique ", "système diacritique " de Merleau-Ponty sont à prendre comme l'équivalent de la proposition de Saussure: "dans la langue il n'y a que des différences sans termes positifs. "Les signifiés " sont purement différentiels, définis non pas positivement par leur contenu, mais négativement par leurs rapports avec les autres termes du système. Leur plus exacte caractéristique est d'être ce que les autres ne sont pas" (Cours, p. 162). Et le signifiant "dans son essence n'est aucunement phonique, il est incorporel, constitué, non par sa substance matérielle, mais uniquement par les différences qui séparent son image acoustique de toutes les autres" (Cours, p. 164). C'est cela que Merleau-Ponty retient de Saussure quand il parle de "système diacritique ". Et des passages comme celui-ci : "la langue ne peut être qu'un système de valeurs pures" (Cours, p. 155). "L'idée de valeur, ainsi déterminée, nous montre que c'est une grande illusion de considérer un terme simplement comme l'union d'un certain son avec un certain concept. Le définir ainsi serait l'isoler du système dont il fait partie : ce serait croire qu'on peut commencer par les termes pour construire le système en faisant la somme, alors qu'au contraire c'est du tout qu'il faut partir pour obtenir par analyse les éléments qu'il renferme." (Cours, p. 157). Quand Merleau-Ponty utilise le terme "système diacritique ", loin d'en faire un "passe-partout théoriquement révolutionnaire ", il rejoint une idée qui a révolutionné effectivement la manière de concevoir et de décrire les langues, idée qui va à l'encontre des présupposés du sens commun et de la linguistique présaussurienne, idée dont s'est inspiré Merleau-Ponty non seulement pour élaborer sa philosophie du langage mais pour repenser toute sa philosophie ${ }^{12}$. Sans doute, Martin va-t-il proclamer que les

12. Martin est plus catholique que le Pape, je veux dire Mounin et Martinet. L'essence du fait linguistique, dit Mounin, "réside dans le concept saussurien de valeur [d'un signe] "constituée uniquement par des rapports et des différences avec les autres termes de la langue " (Cours, p. 163); ce qui fait "la plus exacte caractéristique des valeurs est d'être ce que les autres ne sont pas "(Cours, p. 162). "(G. MOUNIN, Linguistique et philosopbie, p. 7). Les parenthèses carrées sont de Mounin lui-même. Martin s'aperçoit-il que Mounin le très pur dit "valeur d'un signe ". Martin lui reprochera-t-il d'avoir trahi Saussure pour ne pas avoir écrit valeur du signifié du signe? Et c'est Martinet qui écrit : "Pour marquer la cohérence interne de la langue Saussure emploie le terme de système. Les formulations, en la matière, sont très catégoriques: les unités linguistiques sont fondamentalement oppositives et chacune est ce que les autres ne sont pas. "(Encyclopadia Universalis, vol. 14, p. 696). Il n'est pas nécessaire d'être grand clerc pour s'apercevoir 
linguistes n'ont aucune emprise sur ce genre d'entreprise. C'est tout entendu. Mounin, lui, il fallait s'y attendre, trouve que " la lecture de Merleau-Ponty reste interprétative et très déformante, [ qu' ] il accomode Saussure à sa propre phénoménologie " et qu'il soumet le texte saussurien à des "extrapolations très subjectives "13. Martin a fréquenté les milieux où on se félicite d'être réaliste, donc non déformant puisqu'on s'interdit rigoureusement n'est-ce-pas toute " extrapolation subjective "! Merleau-Ponty n'a jamais prétendu à ce réalisme et ce serait lui faire injure que de le lui attribuer. Il pensait avoir mieux à faire : s'inspirer de certaines idées et les faire fructifier de son point de vue à lui. La philosophie n'était pas pour lui un musée où conserver intacts les précieux objets que sont Saussure, la Gestaltpsychologie et que sais-je encore. Les idées de ses prédécesseurs et de ses contemporains intéressaient MerleauPonty dans la mesure où elles le faisaient penser, lui aidaient à comprendre son expérience de la parole et à expliciter le Lebenswelt dans ses multiples dimensions. Pour développer sa philosophie, il utilisait tout ce qui lui tombait sous la main. Il s'inspirait aussi bien de la littérature que de la science et des œuvres de philosophes. Il croyait aussi que "la science achète son exactitude au prix d'une schématisation ". Un philosophe ne peut lui emprunter avec profit que s'il ne cesse de confronter les postulats et les résultats de celle-ci avec " une expérience intégrale » dont il lui revient d'expliciter le sens. Ainsi tout en s'inspirant de la théorie saussurienne de la valeur, il affirmera néanmoins : "Les significations de la parole sont toujours des idées au sens kantien, les pôles d'un certain nombre d'actes d'expression convergents qui aimantent le discours sans être proprement donnés pour leur compte. " (Signes, p. 112). Il se trouvera sûrement des historiens consciencieux qui reprocheront à Merleau-Ponty les " contresens fâcheux " et les " utilisations fautives " aussi bien à l'égard de Kant que de Saussure. Je les laisserai à leurs tourments. La seule question qui m'intéresse vraiment est celle-ci : à travers tous ces énoncés, Merleau-Ponty

que c'est exactement ce que désigne Merleau-Ponty par l'expression "système diacritique ". Notons que Martinet, au lieu d'insister comme Martin sur la proposition que l'union du signifiant au signifié est un fait positif, relève plutôt que l'unité est oppositive et négative : le signe comme total s'oppose aux autres unités et les versants signifiant et signifié de chaque signe ou unité significative ne sont pas ce que sont les autres signifiants et signifiés du système.

13. G. MOUNIN, Saussure, p. 80. 
parvient-il oui ou non à nous faire voir quelque chose de l'expérience de la parole que nous n'avions pas saisi auparavant?

Au moment où j'essaie bien maladroitement, j'en conviens, de ramasser en deux phrases ce qui me paraît l'essentiel du propos de Merleau-Ponty au chapitre "La science et l'expérience de l'expression " de La Prose du Monde, Martin, toujours chaussé de ses lunettes de linguiste-sérieux-rigoureux, laisse tomber : " ce passage ne fait pas beaucoup de sens [sic!! pour un linguiste, parce que d'une part, les mots clefs ne sont pas définis et que d'autre part toutes les affirmations sont autant d'affirmations gratuites. " Et voilà qu'il cite mes deux phrases prenant soin de les décorer de sept points d'interrogation ${ }^{14}$. Je gage qu'avec un peu de bonne volonté il aurait pu économiser sur les points d'interrogation. Ces deux malheureuses petites phrases maintenant trop honorées par une telle fureur interrogative voulaient indiquer à grands traits comment Merleau-Ponty concevait à la fois la décadence et l'émergence des valeurs expressives, des significations, signifiés ou sens autant que des styles. "La volonté d'expression elle-même est ambiguë et renferme un ferment qui travaille à la modifier : chaque langue, dit par exemple Vendryès, est soumise à chaque moment aux besoins jumeaux et contraires de l'expressivité et de l'uniformité. "' ${ }^{15}$ Quelques pages auparavant, se référant à Saussure, (Cours, pp. 122. 123), Merleau-Ponty s'était intéressé au passage du latin au français et au fait que la perte des flexions avait quelque chose à voir avec la place de l'accent en français. "L'accent sur la dernière syllabe est alors retenu comme règle puisqu'il envahit jusqu'aux mots d'emprunt, qui ne devaient rien au latin." (P.M., p. 48). De même à la perte de la flexion a succédé l'emploi généralisé de la préposition et de l'article. Merleau-Ponty se demande : "Comment comprendre le moment fécond de la langue, qui transforme un hasard en raison et d'une manière de parler qui s'effaçait, en

14. J'ai le sentiment que Martin a attrapé ce tic de Mounin qui lui non plus ne lésine pas sur les points d'interrogation quand il veut insinuer que celui qui parle ne sait pas ce qu'il dit. "Aucun linguiste, dit Mounin, ne saurait tirer quelque chose des pages 49-50 de Signes, avec des formules comme celle-ci : "C'est parce que le signe est diacritique [?] c'est parce qu'il se compose et s'organise avec lui-même [?] qu'il a un intérieur [?] et qu'il finit [?] par réclamer [?] un sens. Plus vite les philosophes cesseront de parler du langage de cette manière, et plus vite on pourra rétablir un véritable pont entre philosophie et linguistique. "(G. MOUNIN, Clefs pour la linguistique, p. 11-12).

15. M. MERLEAU-PONTY, La Prose du Monde, p. 50. 
fait soudain une nouvelle, plus efficace, plus expressive, comme le reflux même de la mer après une vague excite et fait grandir la vague suivante?"(P.M., p. 49). Une institution linguistique, comme toute institution d'ailleurs, sera dite vigoureuse ou puissante dans la proportion même où elle permettra des gestes expressifs. Un geste sera dit expressif dans la mesure où il est habité et animé par un style. Un style sera défini comme . . etc., etc ${ }^{16}$. Quand dans mon livre je parlais de relative constance du système phonologique je voulais simplement dire que ce n'est pas tous les jours qu'on abandonne une douzaine de phonèmes pour en créer cinq ou vingt autres. Je disais aussi que la langue impliquait cependant une certaine versatilité ou souplesse, autrement dit que cette institution n'avait pas dans sa dimension sémantique (ou axiologique) la même fixité, la même rigidité et univocité que le code de la route par exemple. Les styles Proust, Valéry, Racine, Eluard ou Rimbaud auraient-ils pu se construire si la langue était un système genre code de la route? Assez de bavardage!

À mon tour d'interroger. Martin affirme que la définition que Martinet propose de la langue doit être considérée " comme un axiome et comme le résultat d'un long processus inductif ". En tant qu'axiome cette définition commande de façon a priori la description de la langue et cette même définition " est le résultat de l'analyse des faits linguistiques, dans la mesure où elle est la conclusion nécessaire à laquelle nous amène la description de la langue ". Que voilà un discours difficile à entendre! Première difficulté : j’ai toujours cru que le processus inductif, si long soit-il, ne conduit jamais à une conclusion nécessaire. Deuxième difficulté : la conclusion nécessaire qu'est la définition est le résultat de l'analyse des faits et pourtant ces faits sont analysés grâce à la définition. Bref, il faut déjà avoir la définition pour analyser correctement les faits. Et on nous dit du même souffle que c'est l'analyse des faits qui nous fournira cette définition.

Entre une conclusion inductive plus ou moins probable et une conclusion nécessaire, la distinction est capitale. S'il faut accorder à Martin que plus de linguistique serait parfois à souhaiter chez le

16. En attendant que je puisse lui fournir toutes les définitions, formalisations et axiomatisations, je prierai Martin de lire l'ouvrage de G.G. GRANGER, Essai d'une philosophie de style, A. Colin. Il trouvera là beaucoup plus de définitions que je pourrais lui en offrir ici. 
philosophe qui parle du langage, serait-il outrecuidant de penser qu'un peu plus d'épistémologie, de logique ou de philosophie des sciences ne serait pas superflu pour les linguistes qui s'aventurent sur des questions comme celle que nous examinons présentement !

Ce que Martin dit du statut de la structure et des relations entre faits et théories ne me convainc aucunement. Il répète simplement Martinet et Mounin à ce sujet. Je n'étais pas d'accord avec eux il y a dix ans, je le suis encore moins aujourd'hui. Ce n'est pas le lieu pour débattre en long et en large ces questions, à mon avis, fondamentales. Déjà dans mon livre, j'avais relevé les thèses de Martinet et je les avais brièvement discutées : "la structure est dans les faits eux-mêmes" dit Martinet ; le "modèle est ce que réalise le chercheur, pour faire comprendre aux autres ce qu'est la structure "; la structure nous est donnée "par une constatation fondée sur l'observation intelligente de cet objet " qu'est la langue, etc., etc. Et Martin de s'exclamer : "le linguiste n'a pas à inventer les faits linguistiques, ils sont là, en quelque sorte sous ses yeux." Comme s'il suffisait, pour faire de la science, d'ouvrir yeux et oreilles et de pratiquer ces "opérations abstractives" dont nous parle Martin à la suite de Mounin ${ }^{17}$. Faut-il répéter encore une fois que ni les "opérations abstractives", ni l'observation, si intelligente soit-elle, ni l'usage des "appareils oscillographiques et cinéradiologiques" ne suffisent à faire d'une connaissance une connaissance scientifique? Alors même que Martin vient d'introduire un peu plus de confusion sur les rapports faits / théories, structure / modèle, il prétend avoir trouvé la réponse. Quant au statut de la linguistique fonctionnelle et structurale de Martinet, je voudrais bien un jour qu'on réponde aux questions suivantes : s'agit-il d'une science empirico-formelle? Et si oui, qu'attend-on pour formaliser ou axiomatiser? C'est le seul moyen qu'on connaisse pour arriver à un peu de rigueur en science. Eux qui se piquent de scientificité et d'objectivité, ils devraient se soucier de problèmes de ce genre. Je voudrais bien savoir aussi comment ils s'y prennent pour tester les hypothèses fondamentales et les propositions qui en déri. vent? Sont-elles confirmables, infirmables, falsifiables? Avant de crier victoire et de se contenter de trouver gratuites les affirmations des autres, je propose à Martin d'examiner la linguistique de Martinet selon les canons des sciences arrivées à maturité. Qu'on lui

17. G. MOUNIN, Linguistique et philosophie, P.U.F., 1975, pp. 8-9. 
administre le traitement que Hempel applique à la physique et à la biologie par exemple ${ }^{18}$. Si la linguistique n'est ni de la philosophie ni de la littérature ni une science formelle, elle est je suppose une science empirique. Une science de l'homme, dira-t-on. Justement, faut-il reconnaître que les sciences de l'homme ne sont pas sciences comme les sciences de la nature, ne sont pas formalisables comme celles-ci, n'obéissent pas aux mêmes canons de rigueur, aux mêmes procédures de mise à l'épreuve des hypothèses et des théories? Martin, lui, se contente de nous annoncer que "les faits linguistiques auxquels se trouve confronté le linguiste demeurent les mêmes peu importe la théorie explicative \%. Ce ne sont pas les exemples qu'il apporte et que $j$ 'ai vus cent fois cités partout qui me convaincront. Comme si la perception ou l'observation des faits, la description des faits se faisaient sans théorie, sans outils conceptuels ${ }^{19}$. Et ces divins appareils qui nous livrent des "faits mesurables objectivement ", les mesurent selon une perspective inscrite en eux par celui qui est à l'origine de leur construction ; et les données fournies par eux sont déchiffrées n'est-ce-pas par quelqu'un qui interprète, c'est-à-dire retient ce qui est pertinent de son point de vue et ignore le reste ${ }^{20}$ ? Personne n'a prétendu que la différence entre pain et bain pour des sujets parlant français relevait purement du rêve ou de l'hallucination. Mais la découverte des phonèmes $/ \mathrm{p} /$ et $/ \mathrm{b} /$, leur description scientifique du point de vue de la phonologie, c'est-à-dire en termes de traits distinctifs ou pertinents est une toute autre histoire. Il ne suffit plus, Martin en conviendra, d'ou-

18. Voir, entre autres, C.G. HEMPEL, Eléments d'épistémologie, A. Colin, Coll. U 2, $1962,184 \mathrm{p}$.

19. Je dirai en passant mon accord le plus total avec cette thèse de $C$. Castoriadis : "Un fait d'expérience n'est tel que dans le cadre d'une théorie ; encore une fois " c'est la théorie qui décide d'abord de ce qui est observable ". Nous ne pouvons donc pas faire semblant de croire qu'il existe un monde de faits en soi, qui sont tels qu'ils sont avant toute interprétation scientifique et indépendamment d'elle, auquel nous comparons des théories pour voir si elles sont ou non falsifiées par lui. "(C. CASTORIADIS, "Science moderne et interrogation philosophique dans Ency. clopadia Universalis, vol. 17, Organum, 1973, p. 55

20. Comme le dit R. Bastide : le savant se veut objectif, "il essaie de se supprimer pour se faire remplacer par des machines; mais on déplace seulement alors le locus de la partition entre le sujet et l'objet, on le situe après, dans l'interprétation des données apportées par la machine, au lieu de le situer avant, dans l'observation empirique. Personnellement j'irai encore plus loin, car on n'expérimente pas sur le réel à travers des instruments, on expérimente à travers la théorie de l'instrument; et cette théorie est une construction de l'esprit humain ". (R. BASTIDE, Préface à G. DEVEREUX, Essais d'ethnopsychiatrie générale, Éd. Gallimard, 1970, p. XVI). 
vrir les yeux et de regarder " ce qui est ", d'observer les faits dans leur réalité ni même de s'adonner à ces inoffensives "opérations abstractives". Le linguiste Prieto lui-même a dénoncé "l'illusion de ceux qui croient pouvoir partir, pour l'étude d'un objet, de "l'expérience vécue", des "faits concrets", ou de quelque chose dans le genre, et non pas, comme c'est inévitable, de la définition de concepts, c'est-à-dire l'explicitation des classes à travers lesquelles cet objet est connu au départ, quel que soit le degré de scientificité de cette connaissance initiale $n^{21}$. Les remarques de Martin sur les instruments qu'utilisent les phonéticiens et sur la façon dont travaillent les phonologues me font penser que le même Prieto nous a offert sur la formation de la phonologie et sur ses relations avec la phonétique instrumentale des renseignements et des réflexions du plus haut intérêt ${ }^{22}$. Le phonologue explique le «sentiment linguistique " du sujet parlant en offrant une description des phonèmes que celui-ci utilise pour se faire comprendre et qu'il sait reconnaître dans le discours qu'il entend. Si la phonétique instrumentale a contribué à la découverte du phonème et à la constitution de la phonologie comme discipline autonome, ce n'est pas parce que "l'emploi d'instruments dans l'étude des sons augmente la capacité de réception sensorielle du sujet et révèle ainsi des caractéristiques qu'on ne saurait en aucun cas reconnaître à l'oreille nue ; au contraire, c'est en révélant la présence dans le son de caractéristiques qu'on peut reconnaître à l'oreille nue, mais que le sujet ignore systématiquement, que la phonétique conduit à cette découverte fondamentale qu'est le phonème ${ }^{23}$. La merveille justement qui permet l'échange entre les sujets parlant la même langue est qu'ils prennent pour le même phonème, c'est-à-dire pour absolument identiques, des "blocs" de bruits ou de sons qui reviennent dans la suite des énoncés, blocs de sons que le phonologue, comme

21. L.J. PRIETO, "La découverte du phonème. Interprétation épistémologique " dans La Pensée, no 148 , déc. 1969 , p. 46. Prieto se dit en désaccord avec la position qui veut que "le seul travail de la connaissance sur l'objet c'est l'abstraction, qui veut que pour connaître l'objet " tel qu'il est réellement " il suffit de reconnaître toutes les caractéristiques qu'il présente et à les distinguer par conséquent de tous les objets dont il est susceptible d'être distingué : bien au contraire - et nous retrouvons notre point de départ - avant que la connaissance ne construise l'objet il n'y a que la totale non-distinction de celui-ci, la totale non-reconnaissance de ses caractéristiques. Le problème que pose toute connaissance est donc celui des distinctions que le sujet fait" (ibid., p. 52).

22. Voir, par exemple, L.J. PRIETO, "Considérations sur la phonologie " dans Études de linguistique et de Sémiologie générales, pp. 153-167.

23. L.J. PRIETO, "La découverte du phonème", p. 52. 
le sujet parlant, admet être membres de la classe que constitue le phonème $/ \mathrm{p} /$ par exemple alors que l'appareil du phonéticien, lui, enregistre ce dont fait " abstraction " le sujet parlant et le phonologue. Que Martin cesse de se torturer! Quand Merleau-Ponty décrit le passage du babillage à la parole et qu'il dit que les phonèmes sont "les vrais fondements de la parole ", il veut simplement dire que la reconnaissance des unités distinctives est condition nécessaire de la compréhension du discours de l'autre et de l'exercice de la parole par le sujet. Reconnaître une identité à une entité quelconque, c'est reconnaître son appartenance à une classe. Et tout classement s'effectue selon un point de vue. Pour la connaissance ordinaire comme pour la connaissance scientifique, il n'y a de fait et d'identité d'un objet qu'en fonction de concepts qui sont autant de principes de classification, d'identification de ce qui se donne à l'expérience, à l'observation, à l'expérimentation. Sans catégorie, sans principe de classification, non seulement pas d'identification possible mais pas de fait ${ }^{24}$.

La position épistémologique de Martin, comme celle de Martinet et de Mounin d'ailleurs, est celle du réalisme. Merleau-Ponty a passé sa vie à chercher une voie entre le réalisme et l'idéalisme. Pour Martinet, la structure est immanente aux faits ; les faits sont là déjà constitués, ils s'imposent à nous. Nous n'avons qu'à les reconnaître pour ce qu'ils sont. Merleau-Ponty, lui, rejette et l'interprétation qui fait de la structure un substitut de l'essence ou de l'idée et l'interprétation qui la considère comme un simple fait innocent de toute catégorie, de tout "modèle " ou de toute théorie. La pensée structuraliste indiquait selon lui un chemin au-delà du primat de l'objet ou du sujet ${ }^{25}$, une voie qui permettrait d'échapper à la pensée objectiviste prise justement entre l'idée pure et le fait brut. Je vois que ni Merleau-Ponty ni moi n'ont réussi à convaincre Martin qui réaffirme avec on ne peut plus de sérénité le réalisme dont, tout comme Merleau-Ponty et bien d'autres, je ne puis me contenter.

24. L.J. PRIETO, Pertinence et pratique, pp. $81-83$.

25. Comme l'écrit J. Vuillemin : "En tout cas, si Merleau-Ponty pensait probablement que toute connaissance objective demeurait illusoire tant qu'elle ignorait l'intervention latente du sujet, il liait cette idée à cette autre que le sujet ne peut se ressaisir comme tel et qu'il est vain d'assigner à la philosophie la tâche de retrouver les actes constituants d'un tel sujet. " (J. VUILLEMIN, "La méthode indirecte de Maurice Merleau-Ponty" dans Critique, XX, 1964, no 211, p. 1016). 
Je ne m'engagerai pas aujourd'hui dans le vieux problème épistémologique des relations sujet-objet dans le mécanisme des connaissances $^{26}$. Bien sûr, on peut de nos jours comme dans l'antiquité et au moyen-âge défendre une épistémologie réaliste. Mais pour ma part, je n'éprouve aucune satisfaction à entendre quelqu'un réaffirmer le réalisme du sens commun sans avoir consenti à se débattre sérieusement avec Kant et le néo-kantisme, avec la phénoménologie et les grands philosophes des sciences du XXe siècle. $S$ 'il n'est pas interdit d'être réaliste en cette fin du XXe siècle, il est à mon avis interdit de l'être comme l'est l'homme de la rue ou comme on pouvait l'être au moyen-âge.

Quant à savoir si l'épistémologie relève de la science ou de la philosophie, c'est là une énorme question. Les réflexions récentes de S. Auroux ${ }^{27}$ à l'occasion d'un ouvrage de Desanti m'ont conduit là où j'en suis présentement. Je pense comme lui que pour le moment au moins "l'épistémologie possède une syntaxe analogue à celle des langues naturelles, que, si elle est science, ce n'est pas au sens courant du terme" (p. 320). Ce qui ne veut pas dire qu'elle est condamnée à coïncider avec la philosophie des sciences traditionnelle.

Il faut distinguer, il me semble, linguistique, philosophie du langage, philosophie de la linguistique (partie de la philosophie des sciences), philosophie linguistique, épistémologie. Ma thèse est qu'un philosophe peut parler du langage sans connaître le moindre mot de la linguistique et élaborer une philosophie du langage aussi valable que quelqu'un qui a toute sa vie pratiqué la linguistique. Tout comme le linguiste peut développer une théorie révolutionnaire sans connaitre le moindre mot des discours philosophiques sur le langage. La philosophie de la linguistique, elle, prend pour objet de réflexion cette discipline qu'est la linguistique. L'ouvrage de Searle ${ }^{28}$, par exemple, en est un de philosophie du langage, non

26. Voir à ce sujet l'étude de J. PIAGET, "Les courants de l'épistémologie scientifique contemporaine "dans Logique et connaissance scientifique, Encyclopédie de la Pléiade, pp. 1225-1271.

27. S. AltrouX, "Qu'est-ce que l'épistémologie?" dans Dialogue, juin 1976, pp 302-320.

28. J.R. SEARLE, Les Actes du langage Essai de Philosophie du langage, Hermann. Paris, Coll. Savoir, 1972. 
de philosophie de la linguistique, ni de philosophie linguistique ${ }^{29}$, ni de linguistique ou d'épistémologie. Il ne se réfère pas à la lin. guistique, science empirique, et il a raison de ne pas le faire puisque sur les questions dont il discute, elle n'a à peu près rien à lui apprendre.

Mounin et Martin souffrent : les philosophes, disent-ils, connaissent mal la linguistique. Là est la source du malheur qu'est le fossé entre savants et philosophes et du dialogue de sourds entre eux. Je constate de mon côté que l'épistémologie n'est pas le fort de l'école de Martinet. Ce qui ne les empêche pas, bien sûr, de se prononcer sur ces questions. Autant leurs travaux de phonologie et de syntaxe m'ont intéressé, autant me désappointent leurs résultats sur la sémantique ou sur l'axiologie ${ }^{30}$ et m'impatientent leurs propos épistémologiques. Les recherches en sémantique d'O. Ducrot me paraissent autrement stimulantes ${ }^{31}$. En ce qui concerne la réflexion épistémologique chez les linguistes, L.J. Prieto me semble aller beaucoup plus loin que Martinet.

La lecture de l'étude critique de Martin m'a apporté une preuve supplémentaire des difficultés de toute entreprise pluri ou interdisciplinaire. C'est toujours à partir d'un lieu et en fonction d'une discipline qui a ses présuppositions qu'on se tourne vers d'autres disciplines. La tâche est bien de trouver un point d'articulation entre les disciplines au-delà du purisme sourcilleux et de la confusion des perspectives.

Université d'Ottawa

29. "Le terme "philosophie linguistique " désigne essentiellement une méthode" " La philosophie linguistique a pour but de résoudre certains problèmes philosophiques particuliers en examinant l'emploi courant de certains mots ou de certains éléments à l'intérieur d'une langue donnée. " (J. SEARLE, Les actes du langage, p. 38).

30. Axiologie, c'est-à-dire étude des valeurs linguistiques. Voir à ce sujet la postface de A. MARTINET, Éléments de linguistique générale, édition de 1974, pp. 208. 210.

31. Voir O. DUCROT, Dire et ne pas dire. Principes de sémantique linguistique, Hermann, Coll. Savoir, 1972, 284 p. ; et aussi O. DUCROT, La preuve et le dire, Repères - Mame, 1973, $290 \mathrm{p}$. 